\title{
Optimal Power Flow Based on Genetic Algorithms and Clustering Techniques
}

\author{
Stefan Stanković and Lennart Söder \\ Department of Electric Power and Energy Systems \\ KTH Royal Institute of Technology \\ Stockholm, Sweden \\ \{stanko, 1sod\}@kth.se
}

\begin{abstract}
Optimal power flow problems have been studied extensively for the past decades. Two approaches for solving the problem have been distinguished: mathematical programming and evolutionary algorithms. The first is fast but is not converging to a global optimum for every case. The second ones are robust but time-consuming. This paper proposes a method that combines both approaches to eliminate their flaws and take advantage of their benefits. The method uses properties of genetic algorithms to group their chromosomes around optima in the search space. The centers of these groups are identified by clustering techniques and furthermore used as initial points for gradient based search methods. At the end, the proposed method finds global optimum and its closest local optima. Continuous Newton-Raphson method is used to overcome ill-conditioned points in search space when calculating power flows. The proposed method is compared against similar methods showing considerable improvement.
\end{abstract}

Index Terms-Continuous Newton-Raphson, Convex Optimization, Genetic Algorithm, K-means, Optimal Power Flow

\section{INTRODUCTION}

Since the first formulation of the optimal power flow (OPF) problem in 1962 by Carpentier [1], a lot of attention has been put on developing fast and robust algorithms for finding the accurate solution. From the beginning, the focus was primarily on computationally-efficient methods that can find an optimal solution in a reasonable time on currently available computer technology. These methods include linear, nonlinear, quadratic programming approaches as well as Newton and interior point methods [2], [3]. All of them can be called under the same name as mathematical programing (MP) methods. OPF are highly nonlinear, non-convex optimization problems with both continuous and discrete control variables. MP methods are not guaranteed to converge to the global optimum for a general OPF problem with multiple local optima [4]. Furthermore, they experience problems with discrete control variables. In order to solve OPF with MP methods, approximations and relaxations of the original problem have to be done leading sometimes to inaccurate solutions.

Evolutionary algorithms (EA) have been recognized as an alternative to MP methods [5]-[10]. They overcome the limitations of MP methods but with the increased computational

This work has been funded by ERA-Net Smart Grids Plus initiative costs. This is one of the reasons that interest in them for solving OPF emerged with advancement of computers at the end of 20th century. The last decade has brought requirements on OPFs to provide more accurate optimal solutions for large scale systems in small amount of time or even real-time. This led to decreased interest in EA because of their poor performance in execution times. Instead, more focus was given on enhancing MP methods to improve their robustness and accuracy while satisfying requirements in speed.

A third group of methods combines previous two to overcome limitations of both and exploit their advantages. One of these hybrid methods [11] uses genetic algorithm (GA) to cope with discrete variables and an interior point method for it's great convergence speed on large scale problems. Another one [12] uses GA to find good initial point for sequential quadratic programming (SQP) optimization method.

This paper proposes a hybrid method that combines GA, clustering techniques and SQP to robustly deliver global and local solutions of OPF with a satisfying speed. It exploits behavior of GA to group its' chromosomes around local optima already in early stages of the algorithm. The main idea is to stop GA when sufficient grouping has occurred allowing extraction of information about the search space. These groups are furthermore identified using clustering techniques. Each cluster of the chromosomes should belong to the convex space surrounding the corresponding local optimum. The optimum can then be found using some of the MP methods where the cluster center is used as the initial point for the search. By simply comparing found local optima, the global optimum is retrieved. In order to ensure convergence of the power flow for all the points in the search space, robust continuous NewtonRaphson method [13] is used.

The proposed algorithm indicates possible improvement in speed among the EA and hybrid methods since it requires less objective function evaluations. Besides the global optimum it also provides information on the closest local optima. Compared to MP methods, it shows better accuracy without significantly larger computational time (up to 7 times) where MATPOWER OPF algorithm [14] is used as a benchmark for the performance analysis. 


\section{Algorithm Structure}

The structure of the proposed algorithm is shown on Fig. 1. Three main parts of the algorithm can be distinguished:

1) genetic algorithm (GA) with robust power flow algorithm,

2) clustering,

3) convex optimization.

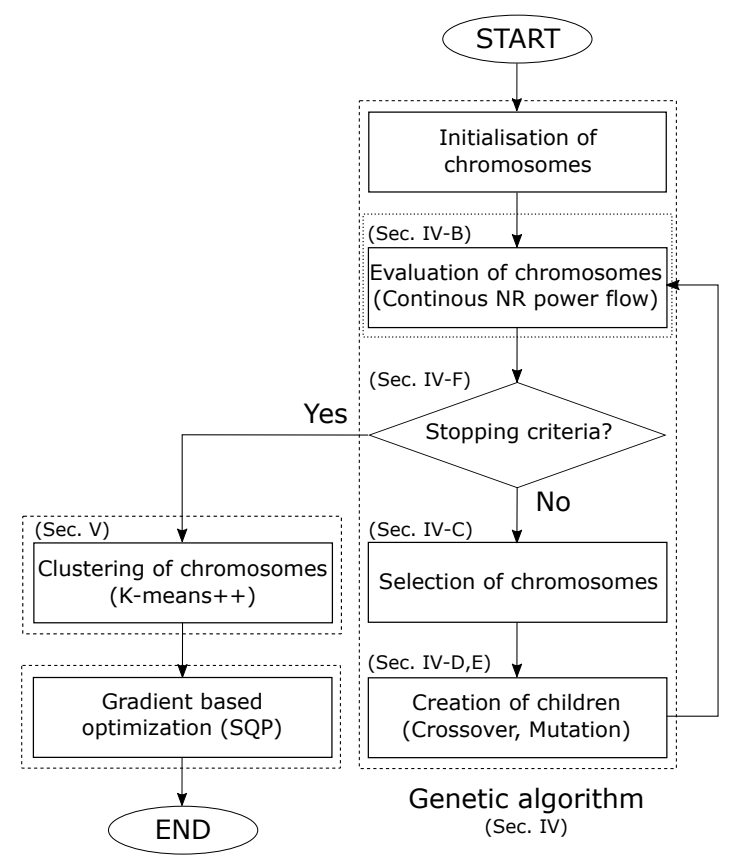

Figure 1. The algorithm flow chart

The algorithm starts by distributing GA's chromosomes uniformly over the search space. This way, without prior knowledge of suitable initial points for the search, the algorithm still keeps in the scope all possible global and local solutions. In order to cope with ill conditioned cases, chromosomes are evaluated by robust power flow algorithm. Fitness (objective) function is calculated from quantities obtained from the power flow. After evaluation of chromosomes, stopping criteria for GA is assessed. It is designed to stop the GA when chromosomes have grouped sufficiently.

After the chromosomes have grouped around optima, these clusters are furthermore identified by clustering algorithm kmeans++. The clusters contain the chromosomes belonging to the same convex area around the corresponding optimum. If the chromosomes are uniformly spread around the optimum, the center of the cluster is exactly that optimum. Otherwise, the optimum can be found using convex optimization methods where the starting point of the search is a cluster center or the fittest chromosome in the cluster.

When all the local optima belonging to different clusters have been found, the global optimum is found simply by taking the local optimum with a highest fitness function. At the end, besides the global optimum, the algorithm provides information on the closest local optima as well. In the following sections each part of algorithm is explained in more detail.

\section{ROBUST POWER FLOW ALGORITHM}

The search space can contain ill-conditioned points of the analyzed system [13], [15], [16]. For these points, standard Newton-Raphson (SNR) power flow algorithm would not converge. The ill-conditioned points are associated with bad initial points that are located outside of the algorithm's basin (region) of attraction. Another reason for non-convergence is the high condition numbers of Jacobian matrix on the trajectory to the solution. High condition numbers make numerical difficulties when trying to find inverse of Jacobian matrix. These usually reflect closeness of solution point to the bifurcation points of the system.

To avoid the mentioned problems, continuous NewtonRaphson (CNR) method [17] is used for calculating power flows. This method makes analogy between update step of SNR and ordinary differential equations (ODE). It furthermore motivates the idea by the fact that the update step of SNR defined in this way can be run using efficient algorithms designed to solve ODE. The recent application on the power flow problem has been presented in [13]. Here, the author uses fourth order Runge-Kutta (RK4) method to integrate the system. Adaptation of the RK4 integration step $\Delta t$ in [13] is done by assessing truncation error $\epsilon$ according to (1). In [16], step size optimization is applied using second order Taylor expansion. It is shown that this method provides better convergence than RK4.

$$
\begin{aligned}
& \text { if } \epsilon>0.01 \text { then } \Delta t=\max (0.985 \cdot \Delta t, 0.75) \\
& \text { if } \epsilon \leq 0.01 \text { then } \Delta t=\min (1.015 \cdot \Delta t, 1.25)
\end{aligned}
$$

Our power flow algorithm consists of two stages:

1) robust CNR stage

2) fast and accurate SNR stage

First stage increases robustness of the algorithm by expanding the basin of attraction so that initial point is inside it. The emphasis in this stage is on robustness with satisfying speed of the algorithm. For this purpose, adaptive time step RungeKutta method (RK23) proposed by Bogacki and Shampine [18] is used in conjunction with CNR. Low order RungeKutta methods such as RK23 are faster than higher order methods but they tend to be less accurate [19]. Accuracy is not very important in the first stage of the algorithm. At this stage, emphasis is on robustness and speed. RK23 has an embedded second-order method. Comparing it's solution with third-order method solution, truncation error $\epsilon$ can be estimated. Integration step $\Delta t$ is adapted starting from initial value $\Delta t_{0}$ according to (2) comparing truncation error of the step with set tolerance $\tau$. Transition to the next stage of the algorithm is done when the operating point is moved in the original basin of attraction of SNR. This is done by assessing the size of the integration step. If it reaches it's maximum value $\Delta t_{\max }$, the algorithm continues to the second stage.

$$
\begin{aligned}
& \text { if } \epsilon>\tau \text { then } \Delta t=\max (0.99 \cdot \Delta t, 0.9) \\
& \text { if } \epsilon \leq \tau \text { then } \Delta t=\min \left(1.01 \cdot \Delta t, \Delta t_{\max }\right)
\end{aligned}
$$


The second stage of the power flow algorithm uses SNR to fast find the accurate solution of the power flow problem. If the assumption that the second stage starts in basin of attraction of SNR is true, the algorithm will converge. Otherwise, it will diverge. To prevent this, SNR step error is assessed. If it starts increasing, the algorithm will return to the first stage. The first stage will put the operating point closer to the solution point before transiting again to the second stage.

Switching heuristics between PU and PQ nodes is implemented from [20]. It is run between each integration step of RK23 in the first stage and each step of SNR in the second stage.

\section{GenetiC ALGORITHM}

We chose GA as a robust search method because of it's great ability to handle nonlinear, non-convex search space that includes discrete variables. Furthermore, it shows great flexibility in the design of a fitness (objective) function allowing it to handle complex multi-objective optimization problems [21], [22]. It has been reported that MP methods do not converge to the global optimum if not appropriately initialized [4]. They tend to get trapped into the local optimum or saddling points of the search space. Deciding about the good initial point usually needs more knowledge about the system and problem itself. GAs as general search methods do not need initial point to start the search. Furthermore, they tend to escape local optima with ease [22]. In our algorithm, we use GA in the first part to extract the information about convex regions of the search space by identifying areas where the chromosomes are grouping.

Multiple derivations of GAs have been developed. The derivation implemented in our algorithm is known as simple GA. These type of GAs are easy to implement and do not require any kind of expert knowledge about the optimization problem. They have three basic evolutionary operators with associated parameters:

1) reproduction $\left(G_{g a p}\right.$-generation gap, change ratio of generation)

2) crossover ( $p_{c}$-probability of crossover)

3) mutation ( $p_{m}$-probability of mutation)

The GA flow is shown on Fig. 1. Each step of our implementation of GA is explained in detail in the following subsections.

\section{A. Coding}

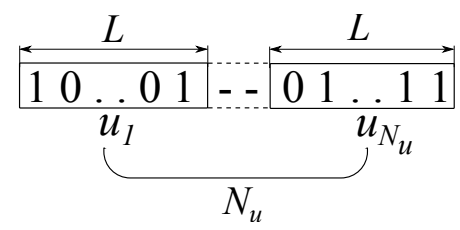

Figure 2. Coding of the chromosomes

For the purpose of our GA we used binary coding of chromosomes. Each controllable variable $u_{i j}$ of chromosome $i$ is represented by $L$ bits or alleles. Minimum value of $u_{i j}$ is coded with integer value 0 while maximum corresponds to integer value $2^{L}-1$. Values in between are coded according to the linear rule. Representation of the controllable variable is called gene. Chromosome is built up from $N_{u}$ genes representing $N_{u}$ controllable variables as shown on Fig. 2.

\section{B. Evaluation of chromosomes - Fitness function}

General expression of a fitness function can be written as:

$$
F_{i}=f\left(X_{i}, U_{i}, \text { par }\right), i=1 . . N
$$

where $N$ is the number of chromosomes. Parameters of the system par are the same for every chromosome. Set of controllable variables $U_{i}$ can be extracted from each chromosome's genome. To calculate the chromosome's fitness function, states of the system $X_{i}$ have to be calculated. They are obtained by running power flow described in Section III that is supplied by $U_{i}$.

Reactive power limits of generating units in the system are accounted by PU-PQ switching heuristics applied in the power flow algorithm. To include limits on other states of the system, penalizing function is introduced:

$$
F_{i}=F_{i}-\sum_{\substack{j=1 \\ x_{j} \notin \Delta x_{j}}}^{M} \delta x_{j} C_{j}, i=1 . . N
$$

where $\Delta x_{j}$ is allowable interval of state $x_{j}, \delta x_{j}$ - absolute value of $x_{j}$ deviation from it's closest limit, $C_{j}$-penalizing factor of state $j$ and $\mathrm{M}$ - number of states in the system. By adjusting $C_{j}$, the limit on state $j$ can be softer or harder.

\section{Selection of chromosomes}

To create a new generation of chromosomes, part of the chromosomes $\left(\left[N\left(1-G_{\text {gap }}\right)\right]\right)$ will be selected to be transfered to the next generation while the rest of generation $\left(\left[N G_{g a p}\right]\right)$ will be filled with the offspring. This ratio is determined by parameter $G_{g a p} \in(0,1)$ called generation gap.

The selection process is done using Roulette wheel technique. Roulette wheel technique selects the chromosomes with probability proportional to the value of their fitness function. Instead of using it directly in the selection process, the raw fitness function $F_{i}$ is firstly appropriately scaled to enhance GA's convergence. The scaling methods described in [21], [22] are used:

1) linear scaling

2) power scaling

3) sigma scaling (truncation)

Before applying Roulette wheel, it must be assured that values of fitness function for each chromosome are positive. This is achieved by linear scaling of the fitness function $F_{i}$ using (5). Coefficient $a$ is readjusted for each generation in such way that $\min \left(F_{1}\right)=0.02 \max \left(F_{1}\right)$.

$$
F_{1 i}=a+b F_{i}
$$


After applying linear scaling, the fitness surface might still have low steepness. This will result in slow convergence in later stages of GA. To speed up GA, power scaling (6) is used in the next step. Parameter $k$ has to be tuned depending on how much steeper the surface has to be.

$$
F_{2 i}=F_{1 i}{ }^{k}
$$

Drawback of power scaling is that it might provoke premature convergence of GA. If it occurs, the GA might end up finding local optimum providing a wrong solution. To avoid it, in third step of fitness scaling, sigma scaling is applied. This method exploits statistical information about generation $t$ : mean value of fitness function $\bar{F}_{2}(t)$ and standard deviation $\sigma\left(F_{2}(t)\right)$. Using (7), fitness function is scaled to avoid the problem of premature convergence.

$$
F_{3 i}=1+\frac{F_{2 i}-\bar{F}_{2}(t)}{2 \sigma\left(F_{2}(t)\right)}
$$

After scaling fitness function $F_{i}, F_{3 i}$ is obtained and used in roulette wheel selection process.

\section{Crossover}

The new generation of chromosomes is completed by adding $\left[N G_{\text {gap }}\right]$ new chromosomes coming from an offspring. To create the offspring, parents from the previous generation are chosen using the same selection procedure described in the previous subsection.

After selection of two chromosomes $i$ and $k$ from previous generation $t$, multi-point crossover is applied to create two children chromosomes (Fig. 3). Multi-point crossover is done by combining single crossover between genes representing the same control variable. Therefore, total number of crossover points is $2 N_{u}-1$. Single crossover points applied to the separate genes $\left(s_{1} . . s_{N_{u}}\right)$ are chosen randomly for the each

\begin{tabular}{|c|c|c|c|c|c|}
\hline \multicolumn{2}{|r|}{$s_{1}$} & $s_{2}$ & & $S_{N_{u^{-1}}}$ & \multirow[t]{2}{*}{$S_{N_{u}}$} \\
\hline & & \multicolumn{2}{|c|}{ parents } & & \\
\hline$i$ & \begin{tabular}{ll|ll}
00 & 01 & 1 & 01 \\
\end{tabular} & 111 & $\begin{array}{lllllllllll}0 & 1 & 0\end{array}$ & \begin{tabular}{lll|lll}
1 & 0 & 1 & 0 & 1 & 0
\end{tabular} & $\begin{array}{lllllllllll}0 & 1 & 1\end{array}$ \\
\hline \multirow[t]{3}{*}{$h$} & \begin{tabular}{|l|llll|lllll}
0 & 0 & $\ldots$ & 1 & 0 & 1 & 1 & 0
\end{tabular} & 100 & 111 & \begin{tabular}{|lll|llll}
0 & 0 & 0 & 0 & 0 \\
\end{tabular} & 1110 \\
\hline & & \multicolumn{2}{|c|}{ children } & & \\
\hline & 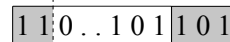 & 100 & 010 & \begin{tabular}{lll|lll}
0 & 0 & 1 & 0 & 1 & 0
\end{tabular} & 110 \\
\hline & \begin{tabular}{|l|lllll|lll|}
0 & 0 & $\ldots$ & 0 & 0 & 1 & 1 & 1 & 0 \\
\end{tabular} & 111 & 111 & \begin{tabular}{lll|llll}
1 & 0 & 0 & 0 & 0
\end{tabular} & 011 \\
\hline
\end{tabular}
pair of parents.

Figure 3. Creation of children

\section{E. Mutation}

Operation of mutation is done on random alleles in random chromosomes by changing their bit value. Total number of mutated alleles in generation is $N_{a l} p_{m}$ where $p_{m}$ is probability of mutation and $N_{a l}=L N_{u} N$ is total number of alleles in the generation.

\section{F. Stopping criteria}

GA is supposed to stop it's search when it reaches satisfying convergence level or maximum number of generations [21], [22]. Unfortunately, this goal can lead to substantial computational time of the algorithm. The goal of GA in our algorithm is to substantially group chromosomes around global optimum and closest local optima so that groups can be identified. Therefore, first condition is applied with very relaxed threshold in the form of (8) where $\sigma(F)$ is standard deviation of chromosomes' fitness functions and $\bar{F}$ is the mean value. The second condition of maximum number of generations for which GA is allowed to run is set to relatively low number to avoid excessive computational times in case when the first criteria has not been met.

$$
\frac{\sigma\left(F_{1}\right)}{\bar{F}_{1}}<0.2
$$

\section{G. Parallel Computing}

The main drawback of GAs comes from the big computational time they take to converge. Fortunately, they are very suitable to be used with parallel computing [23]. In our algorithm, parallel computing is applied when evaluating fitness functions of chromosomes.

\section{Clustering of Chromosomes}

GAs are recognized as very robust method efficient in finding global optimum when search space is complex. Their main disadvantage comes from the long time they take to converge. The algorithm we are proposing uses GA to extract the information about the search space. The goal is to divide the non-convex search space into it's convex parts surrounding global and local optimums. The GA is run for a number of generations to allow sufficient grouping of chromosomes around optimums in the search space. These groups are then identified using clustering technique k-means.

$\mathrm{K}$-means as a clustering technique was first proposed by Lloyd in [24]. It groups the data points in the space in $k$ clusters such that the sum of squared Euclidean distances between each point and it's closest cluster center is minimized. The algorithm starts by randomly initiating the clusters' centers. The enhanced cluster center initialization is proposed in [25]. Resulting algorithm is known as k-means++. For the purpose of our OPF, k-means++ algorithm realization proposed by Hamerly in [26] is used. To determine the number of clusters $k$, elbow method is applied.

The last generation that satisfied the stopping criteria of GA is used as the dataset $D$ to be clustered. Each chromosome $i$ is represented in $D$ with a data point $d_{i}$ :

$$
d_{i}=\left[\hat{U}_{i} ; \hat{F}_{2 i}\right]
$$

where $\hat{U}_{i}$ is a set of controllable variables normalized to the range $\hat{u}_{i j} \in \alpha_{j}[0,1], j=1 . . N_{u} . \hat{F}_{2 i}=\beta F_{2 i} / \max \left(F_{2}\right)$ is normalized fitness function of chromosome $i$. Coefficients $\alpha_{j}$ and $\beta$ account for strength of clustering in certain dimension. With lower value of coefficient $\alpha_{j}$, effect of clustering on 
dimension $j$ is decreased since the projection of data points on that dimension will be smaller. With certain expert knowledge about the OPF problem, the coefficients can be chosen to enhance the clustering.

\section{GlobAl AND CLOSEST LOCAL OPTIMA}

When the clustering is done, the centers of clusters should be located in convex subspaces surrounding the optima. Cluster centers will be in the same position as these optima if the chromosomes have distributed uniformly around optima. For most of the scenarios, this is not the case. The optimum can then be found using some of the convex optimization strategies where for the initial point of search, cluster center is used. This is possible since the cluster center and it's associated optimum are in the same convex part of the search space. In most of the cases, the center will be relatively close to the optimum resulting in fast convergence of convex optimization. The global optimum is found by comparing the values of fitness functions of all identified optima.

To show the performance of the proposed algorithm, OPF is applied on IEEE14 bus system. The goal is to find maximum consumption of the reactive power from the slack bus 1 by the IEEE14 grid while minimizing the active power losses in the grid. This example illustrates the arising question in scientific community about using distribution grids and distributed generation to help with voltage control on transmission grid [27]-[29]. Here, IEEE14 is used as a distribution grid that provides support to a transmission grid (slack bus 1).

The optimization problem is mathematically expressed as (10). The multi-objective function illustrates the trade-off between supplied (consumed) reactive power $Q_{1}$ and active power losses in the system $\sum \Delta P$. The goal is to maximize the profit having in mind that $A_{1}$ reflects the price of remuneration per $M V A r h$ and $A_{2}$ reflects the price of $M W h$. The physical properties of the system are accounted through constraints. Equality constraints consist of active $P_{i n j_{i}}$ and reactive $Q_{i n j_{i}}$ power balance equations. Inequality constraints reflect on voltage limits $\left[U_{\min _{i}} U_{\text {max }_{i}}\right]$ where Bus is set of all buses in the system. Reactive power limits of generators $\left[Q_{\min _{j}} Q_{\text {max }_{j}}\right]$ in set Gen are accounted by last inequality in 10 .

$$
\begin{array}{ll}
\text { obj. } & \max \left(A_{1} Q_{1}-A_{2} \sum \Delta P\right) \\
\text { s.t. } & \sum P_{\text {inj }_{i}}=0 \\
& \sum Q_{i n j_{i}}=0 \\
& U_{\text {min }_{i}}<U_{i}<U_{\text {max }_{i}}, \\
& Q_{\text {min }_{j}}<Q_{j}<Q_{\text {max }_{j}}, \\
& \forall i \in \text { Bus, } \forall j \in \text { Gen }
\end{array}
$$

Parameters of the proposed OPF algorithm are listed in Table I. SQP is chosen as convex optimization algorithm. The fitness function coefficients have values: $A_{1}=1.2$ and $A_{2}=60$. Controllable variables are chosen to be voltages of

\begin{tabular}{|c|c|c|c|}
\hline & Parameter & Symbol & Value \\
\hline \multirow{3}{*}{ 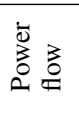 } & Initial time step & $\Delta t_{0}$ & 1.1 \\
\hline & Maximum time step & $\Delta t_{\max }$ & 1.111 \\
\hline & Runge-Kutta tolerance & $\tau$ & 0.0001 \\
\hline \multirow{10}{*}{ 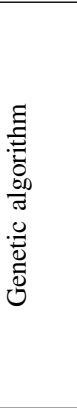 } & Number of chromosomes & $N$ & 400 \\
\hline & Number of genes & $N_{u}$ & 2 \\
\hline & Number of alleles & $L$ & 12 \\
\hline & Generation gap & $G_{\text {gap }}$ & 0.7 \\
\hline & Probability of crossover & $p_{c}$ & 0.8 \\
\hline & Probability of mutation & $p_{m}$ & 0.0008 \\
\hline & Maximum number of generations & $N_{g e n_{\max }}$ & 15 \\
\hline & Fitness penalizing coefficients & $C_{j}, j=1 . . M$ & $10^{8}$ \\
\hline & Fitness linear scaling coefficient & $b$ & 1 \\
\hline & Fitness power scaling coefficient & $k$ & 5 \\
\hline \multirow{2}{*}{ 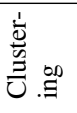 } & Clustering coefficient & $\alpha_{j}, j=1 . . N_{u}$ & 1 \\
\hline & Clustering coefficient & $\beta$ & 0.5 \\
\hline
\end{tabular}
generators at buses:
- case 1: bus 3 and 6 ,

- case 2: bus 3 and 8 .

TABLE I

PARAMETERS OF THE OPF ALGORITHM

Contour plot of search space with results for case 1 is shown on Fig. 4(a) and for case 2 on Fig. 4(b). Identified cluster centers are shown with black asterisks, red pluses are local optima while the global optimum is represented by black plus. For better data visualization, scaled fitness function $F_{3}$ is used on z-axis.

For the case one, it can be seen that the chromosomes have grouped around two optima in the search space. Clustering algorithm has decided, using elbow method, to divide the space into three groups. Therefore, the group around the global optimum is split into two clusters. This will not create any problems since both cluster centers will converge to the same optimum. On the other hand if the threshold in Elbow method has been set towards choosing smaller number of clusters, the problems could have occurred resulting in possible wrong final solution. Therefore, the threshold is set towards choosing larger number of clusters to improve robustness of the method.

In the case two, the space is again divided into three clusters. But now, each cluster belongs to one optimum in the search space: global optimum, one local optimum and one saddling point. The saddling point is not retrieved by the algorithm since the SQP has successfully passed it going from the cluster center towards the local optimum.

Both of the cases show examples of our algorithm's ability to retrieve two close optima in the non-convex search space and find which one is the global optimum.

\section{ViI. Performance analysis}

Previous section has illustrated ability of our algorithm to cope with non-convex search space and identification of the global optimum. This section will provide comparative analysis of our algorithm with the popular algorithm for calculating power flows and OPFs - MATPOWER [14]. Both 


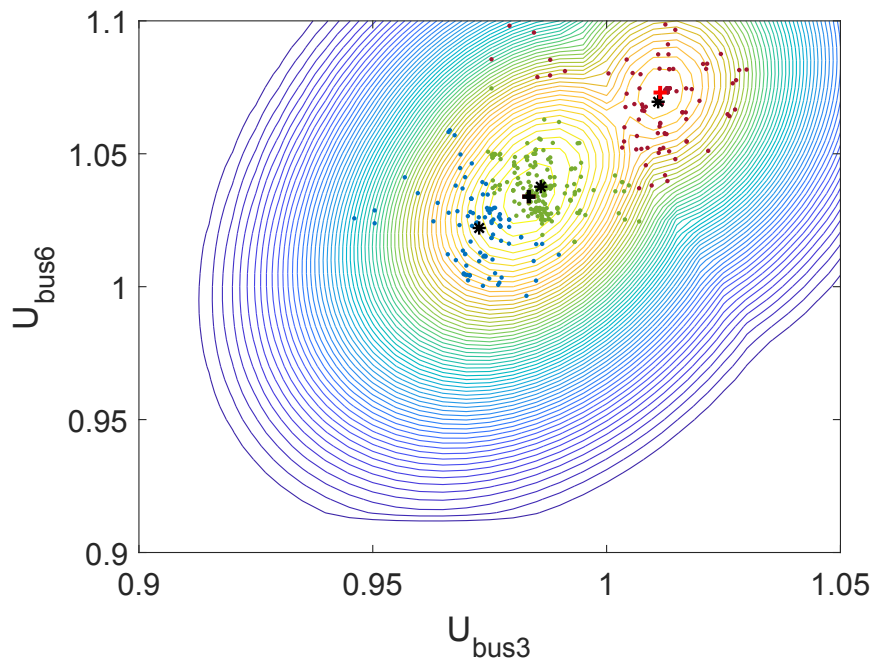

(a) Case 1

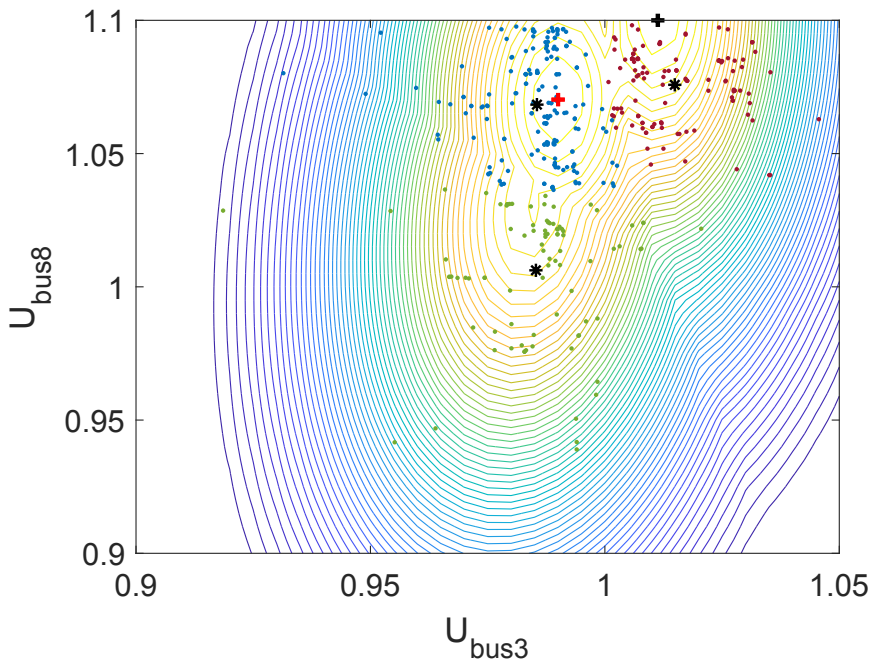

(b) Case 2

Figure 4. Contour plot of search space with identified cluster centers and optima

algorithms are executed in MATLAB R2017a environment on Windows 7 using Intel Core i7-6600U processor. The comparison is done on modified IEEE 9 bus system [4] for the optimal dispatch problem. This system is reported to have three local and the global solution of the problem.

MATPOWER is initialized with default settings where MATPOWER internal-point solver (MIPS) version 1.2.2 is used to find the solution. After running the MATPOWER OPF algorithm, the results provided in Table II are obtained. MATPOWER found the result in 1.03 seconds. Comparing them with reported results in [4] it can be seen that MATPOWER converged to the one of the local optima.

TABLE II

RESULTS OF THE MATPOWER OPF FOR MODIFIED IEEE 9 BUS SYSTEM

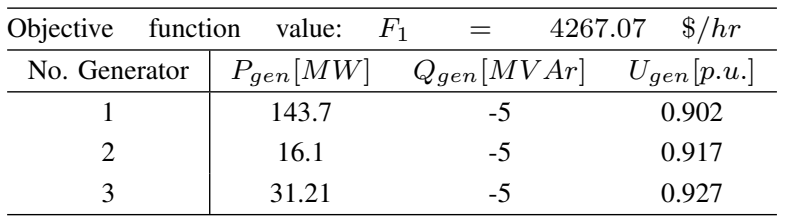

Our OPF algorithm is initialized with slightly modified parameters than for the case in the previous section. Differences in parameters are listed in Table III. As control variables, generator voltages $U_{\text {gen }}$ and active powers $P_{\text {gen }}$ are chosen. Active power of slack generator 1 is excluded from control variables since it is dependent on values of active powers of two other generators. Therefore, first three genes represent voltages of the generators while the last two represent active powers of generator 2 and 3 . For this case, fitness power scaling coefficient $k$ is set to 0.5 to additionally lower the chance of premature convergence of GA. Clustering coefficients are chosen to enforce clustering in the active power dimensions of the search space. Using our algorithm, the results provided in Table IV are obtained in 6.94 seconds.
TABLE III

DIFFERENCES IN PARAMETERS OF THE PROPOSED OPF ALGORITHM

\begin{tabular}{|c|c|c|c|}
\hline & Parameter & Symbol & Value \\
\hline \multirow{3}{*}{ 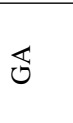 } & Number of genes & $N_{u}$ & 5 \\
\hline & Fitness linear scaling coefficient & $b$ & -1 \\
\hline & Fitness power scaling coefficient & $k$ & 0.5 \\
\hline \multirow{3}{*}{ 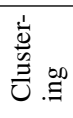 } & Clustering coefficient & $\alpha_{j}, j=1 . .3$ & 0.1 \\
\hline & Clustering coefficient & $\alpha_{j}, j=4 . . N_{u}$ & 1 \\
\hline & Clustering coefficient & $\beta$ & 0.8 \\
\hline
\end{tabular}

TABLE IV

RESULTS OF THE PROPOSED OPF FOR MODIFIED IEEE 9 BUS SYSTEM

\begin{tabular}{|c|c|c|c|}
\hline Optimum 1 & \multirow{2}{*}{\multicolumn{3}{|c|}{$\begin{array}{lcr}\text { Obj. function } & \text { value: } F_{1}=4265.3 \$ / h r \\
P_{g e n}[M W] & Q_{g e n}[M V A r] & U_{g e n}[p . u .]\end{array}$}} \\
\hline No. Generator & & & \\
\hline 1 & 142.25 & -5 & 0.9027 \\
\hline 2 & 10 & -5 & 0.9169 \\
\hline 3 & 38.75 & -5 & 0.9272 \\
\hline Optimum 2 & \multicolumn{3}{|c|}{ Obj. function value: $F_{2}=3087.8 \$ / \mathrm{hr}$} \\
\hline No. Generator & \multicolumn{2}{|c|}{$P_{g e n}[M W] \quad Q_{g e n}[M V A r]$} & $U_{g e n}[p . u]$. \\
\hline 1 & 9.57 & -5 & 0.9094 \\
\hline 2 & 124.89 & -5 & 0.9220 \\
\hline 3 & 57.94 & -5 & 0.9389 \\
\hline Optimum 3 & \multicolumn{3}{|c|}{ Obj. function value: $F_{3}=4246.7 \$ / \mathrm{hr}$} \\
\hline No. Generator & $P_{g e n}[M W]$ & $Q_{g e n}[M V A r]$ & $U_{g e n}[p . u]$. \\
\hline 1 & 143.22 & -5 & 0.9020 \\
\hline 2 & 37.81 & -5 & 0.9175 \\
\hline 3 & 10 & -5 & 0.9247 \\
\hline
\end{tabular}

Comparing the results from Tables II and IV with the ones provided by [4], it can be seen that our algorithm has found the global optimum (Optimum 2, Table IV) and two other local optima. One of these local optima is the same one found by MATPOWER (Optimum 1, Table IV) with the difference that our algorithm provides better precision. MATPOWER could 
possibly give better results with better choice of the initial point. Our algorithm comes with an advantage that it does not need an initial point at all. But, our algorithm takes 7 times more time than MATPOWER to find the solution.

\section{DISCUSSION}

Combination of GAs and clustering techniques proposed in this paper is used to identify convex regions of the search space containing only one optimum. In the cases of very complex search space, it can happen that identified regions are not convex and contain multiple optima. If this is the case, our algorithm will find global optimum in that region, while the local ones will not be identified. This is the cause why our algorithm was not able to find all four optima in modified IEEE 9 bus case.

For the future work, the identified regions can be used to initialize demes of distributed genetic algorithm (DGA). After letting the DGA to progress for certain amount of generations, the demes can be clustered allowing more detailed exploration of the search space. Depending on the scale of the problem, the process can be repeated a couple of times.

Although, our algorithm is slower than MATPOWER MIPS, it shows better robustness and precision when finding global optimum. Compared with other EA and hybrid methods, it has less objective function evaluations indicating probable smaller execution times.

\section{Conclusions}

This paper proposes the new algorithm to solve a general OPF problem. It uses combination of GA, k-means++ clustering algorithm and SQP to identify optima in the search space. Information about the search space is extracted by identifying grouping of chromosomes of GA using k-means++. These groups can be considered as convex regions surrounding optima. After the regions have been identified, SQP algorithm is used to find the local optimum belonging to each of the convex regions. Global optimum is found by simply comparing all identified optima.

The proposed algorithm has been tested on three different OPF problems. While MATPOWER was not able to identify the global optimum on the modified IEEE 9 bus case, our algorithm has found it. Furthermore it identified also two other local optima, one being exactly the one found by MATPOWER.

The approach for solving an OPF problem presented in this paper brings new insights on how combination of GAs, clustering techniques and MP methods can be used to accurately identify global and local optima in the search space.

\section{REFERENCES}

[1] J. Carpentier, "Contribution a l'etude du dispacting economique," Bull. Soc. Francaise des Electriciens, vol. 3, pp. 431-447, 1962.

[2] J. A. Momoh, R. Adapa, and M. E. El-Hawary, "A review of selected optimal power flow literature to 1993. part i: Nonlinear and quadratic programming approaches," IEEE Trans. Power Syst., 1991.

[3] J. A. Momoh, M. E. El-Hawary, and R. Adapa, "A review of selected optimal power flow literature to 1993. part ii: Newton, linear programming and interior point methods," IEEE Trans. Power Syst., 1999.
[4] W. A. Bukhsh, A. Grothey, K. I. M. McKinnon, and P. A. Trodden, "Local solutions of the optimal power flow problem," IEEE Trans. Power Syst., vol. 28, no. 4, November 2013.

[5] M. Todorovski and D. Rajicic, "An initialization procedure in solving optimal power flow by genetic algorithm," IEEE Trans. Power Syst., 2006.

[6] Z. Liu, F. Wen, and G. Ledwich, "Optimal siting and sizing of distributed generators in distribution systems considering uncertainties," IEEE Trans. Power Syst., 2011.

[7] M. S. Kumari and S. Maheswarapu, "Enhanced genetic algorithm based computation technique for multi-objective optimal power flow solution," Int. J. of Elect. Power and Energy Syst., 2010.

[8] A. A. A. E. Ela, M.A.Abido, and S. Spea, "Optimal power flow using differential evolution algorithm," Elect. Power Syst. Res., 2010.

[9] A. G. Bakirtzis, P. N. B. amd C. E. Zoumas, and V. Petridis, "Optimal power flow by enhanced genetic algorithm," IEEE Trans. Power Syst., 2002.

[10] V. A. Evangelopoulos and P. S. Georgilakis, "Optimal distributed generation placement under uncertainties based on point estimate method embedded genetic algorithm," IET Generation, Transmission and Distribution, 2014.

[11] W. Yan, F. Liu, C. Y. Chung, and K. P. Wong, "A hybrid genetic algorithm-interior point method for optimal reactive power flow," IEEE Trans. Power Syst., 2006.

[12] K.-H. Kim, J.-K. Lee, S.-B. Rhee, and S.-K. You, "Security constrained opf by hybrid algorithms in interconnected power systems," in IEEE PES Summer Meeting, July 2001.

[13] F. Milano, "Continuous newton's method for power flow analysis," IEEE Trans. Power Syst., 2009.

[14] R. D. Zimmerman, C. E. Murillo-Sanchez, and R. John, "Matpower: Steady-state operations, planning and analysis tools for power systems research and education," IEEE Trans. Power Syst., vol. 26, pp. 12-19, 2011.

[15] Y. Wang, L. C. P. da Silva, W. Xu, and Y. Zhang, "Analysis of illconditioned power-flow problem using voltage stability methodology," IEE Proceedings - Generation, Transmission and Distribution, 2001.

[16] J. F. Gutierrez, M. F. Bedrinana, and C. A. Castro, "Critical comparison of robust load flow method for ill-conditioned systems," in IEEE Trondheim PowerTech, June 2011.

[17] M. K. Gavurin, "Nonlinear functional equations and continuous analogies of iterative methods," Izv. Vuzov. Ser. Mathematika, 1958.

[18] P. Bogacki and L. F. Shampine, "A 3(2) pair of runge - kutta formulas," Applied Mathematics Letters, 1989.

[19] M. E. Hosea and L. F. Shampine, "Efficiency comparison of methods for integrating odes," Comput. \& Mathematics with Appl., pp. 45-55, September 1994.

[20] J. Zhao, H.-D. Chiang, H. Li, and P. Ju, "On pv-pq bus type switching logic in power flow computation," in 16th PSCC, Galsgow, Scotland, July 2008.

[21] M. Melanie, An Introduction to Genetic Algorithms. MIT Press, 1996.

[22] S. N. Sivanandam and S. N. Deepa, Introduction to Genetic Algorithms. Springer, 2008.

[23] A. Munawar, M. Wahib, M. Munetomo, and K. Akama, "A survey: Genetic algorithms and the fast evolving world of parallel computing," in The 10th Int. Conf. on High Comp. and Comm., 2008.

[24] S. P. Lloyd, "Least squares quantization in pcm," IEEE Trans. Inf. Theory, vol. 28, no. 2, pp. 129-137, March 1982.

[25] D. Arthur and S. Vassilvitskii, "k-means++: The advantages of careful seeding," in 18th апnu. ACM-SIAM symp. on Discrete algorithms, 2007.

[26] G. Hamerly, "Making k-means even faster," in SIAM Int. Conf. on Data Mining, April 2010.

[27] Y. Christayakov, E. Kholodova, K. Netreba, A. Szabo, and M. Metzger, "Combined central and local control of reactive power in electrical grids with distributed generation," in 2nd IEEE ENERGYCON Conf. \& Exhibition / Future Energy Grids and Systems Symp., 2012.

[28] S. Auer, F. Steinke, W. Chunsen, A. Szabo, and R. Sollacher, "Can distribution grids significantly contribute to transmission grids' voltage managment?" in IEEE PES Innovative Smart Grid Technologies Conf. Europe (ISGT-Europe), 2016.

[29] P. Cuffe and A. Keane, "Voltage responsive distribution networks: Comparing autonomous and centralized solutions," IEEE Trans. Power Syst., vol. 30, no. 5, September 2015. 